\title{
A NEW STOCHASTIC EQUIVALENT LINEARIZATION IMPLEMENTATION FOR PREDICTION OF GEOMETRICALLY NONLINEAR VIBRATIONS
}

\author{
Alexander A. Muravyov ${ }^{*} \Gamma$ Travis L. Turner ${ }^{\dagger} \Gamma$ Jay H. Robinson ${ }^{\ddagger} \Gamma$ Stephen A. Rizzi ${ }^{\S}$ \\ NASA Langley Research CenterTStructural Acoustics BranchГHamptonГVAГ23681
}

\begin{abstract}
$\underline{\text { Abstract }}$
In this paperTthe problem of random vibration of geometrically nonlinear MDOF structures is considered. The solutions obtained by application of two different versions of a stochastic linearization method are compared with exact (F-P-K) solutions.

The formulation of a relatively new version of the stochastic linearization method (energy-based version) is generalized to the MDOF system case. AlsoTa new method for determination of nonlinear stiffness coefficients for MDOF structures is demonstrated. This method in combination with the equivalent linearization technique is implemented in a new computer program.

Results in terms of root-mean-square (RMS) displacements obtained by using the new program and an existing in-house code are compared for two examples of beam-like structures.
\end{abstract}

\section{$\underline{\text { Introduction }}$}

Resurgent interest in high speed fight vehicles and the daily operation of the aging commercial and military aircraft fleets necessitate the further development of sonic fatigue technology to understand the fatigue mechanisms and to estimate the service life of aerospace structures subjected to intense acoustic and thermal loads. Efforts to extend the performance and flight envelope of high speed aerospace vehicles have resulted in structures which may behave in a geometrically nonlinear fashion to the imposed loads. Such behavior can have a significant effect on fatigue life. Further improvements in vehicle performance and system design are hampered by the limited understanding of the physical nature of geometrically nonlinear

*NRC Postdoctoral Research Associate

$\dagger$ Aerospace Engineer TMember AIAA

¥Aerospace Engineer

$\S$ Aerospace Engineer $\mathrm{C}$ Associate Fellow AIAA

"Copyright (C) 1999 by the American Institute of Aeronautics and Astronautics Inc. No copyright is asserted in the United States under Title 17TU.S. Code. The U.S. Government has a royalty-free license to exercise all rights under the copyright claimed herein for Governmental Purposes. All other rights are reserved by the copyright owner." structural response. Conventional (linear) prediction techniques can lead to grossly conservative designs and provide little understanding of the nonlinear behavior. A large body of work exists on the prediction of geometrically nonlinear dynamic response of structures. All methods currently in use are typically limited by their range of applicability or excessive computational expense.

Methods currently in use to predict geometrically nonlinear dynamic structural response include perturbation $\Gamma$ Fokker-Plank-Kolmogorov (F-P-K) $\Gamma$ Monte Carlo simulation and stochastic linearization techniques. Perturbation techniques are limited to weak geometric nonlinearities. The F-P-K approach ${ }^{1,2}$ yields exact solutions $\Gamma$ but can only be applied to simple mechanical systems. Monte Carlo simulation is the most general method Tbut computational expense limits its applicability to rather simple structures. Finally stochastic linearization methods (e.g. equivalent linearization $\Gamma \mathrm{see}^{2-6}$ ) have seen the most broad application for prediction of geometrically nonlinear dynamic response because of their ability to accurately capture the response statistics over a wide range of response levels while maintaining a relatively light computational burden.

Implementations of stochastic linearization have been limited to special purpose computer codes until recently when the method of equivalent linearization was introduced into MSC/NASTRAN as a Direct Matrix Abstraction Program (DMAP) Alter ${ }^{7}$. In this study an alternative approach to the solution of nonlinear vibration problems is developed and an independent in-house code based on this approach is implemented.

\section{Equivalent Linearization Techniques}

Two versions of the equivalent linearization technique are considered. One is based on minimization of the error in the force-vector $\Gamma$ and the other minimizes the error in potential energy.

\section{Force Error Minimization Version}

Consider a MDOFTviscously damped linear system. The equations of motion governing such a system can be written in the form

$$
M \ddot{X}+C \dot{X}+K X=F
$$


where $M$ is the mass matrix $\Gamma$ is the damping ma$\operatorname{trix} \Gamma K$ is the stiffness matrix $\Gamma X$ is the displacement response vector and $F$ is the force excitation vector.

For geometrically nonlinear problems of deformation e.g. large deflection flexural vibration of thin plate structures $\Gamma$ the governing equation(s) of motion will include a nonlinear force term $\Gamma(X)$ Ti.e.

$$
M \ddot{X}+C \dot{X}+K X+\Gamma(X)=F
$$

where the vector function $\Gamma(X)$ generally includes 2 nd and 3rd order terms in $X$. There exist mathematical difficulties in the derivation of a general solution to equation (1) for the case of random excitation. An approximate solution can be achieved by formation of an equivalent linear system:

$$
M \ddot{X}+C \dot{X}+\left(K+K_{e}\right) X=F
$$

where $K_{e}$ is the equivalent linear stiffness matrix.

The method of equivalent linearization seeks to minimize the difference between the nonlinear force and the product of the equivalent linear stiffness and displacement response vector. The equivalent linear stiffness satisfying this requirement can be determined from the following condition:

$$
\text { error }=E\left[\left(\Gamma(X)-K_{e} X\right)^{T}\left(\Gamma(X)-K_{e} X\right)\right] \rightarrow \min
$$

where $E[\ldots]$ represents the expectation operator. The latter equation will be satisfied if

$$
\frac{\partial(\text { error })}{\partial K_{e i j}}=0 \quad i, j=1,2, \ldots, N
$$

In this paper $\Gamma$ consideration is limited to the case of GaussianT zero-mean excitation and response to simplify the solution. Omitting intermediate derivations $\Gamma$ the final form for the equivalent linear stiffness matrix becomes (see for example Roberts et al. ${ }^{3} \Gamma$ Atalik et al. $\left.{ }^{4}\right)$ :

$$
K_{e}=E\left[\frac{\partial \Gamma}{\partial X}\right]
$$

\section{Potential Energy Error Minimization Version}

Elishakoff et al. ${ }^{5,6}$ proposed another stochastic linearization approach $\Gamma$ based on potential energy error minimization and numerical results were demonstrated for the case of SDOF systems. In this paperT that approach is generalized for the case of MDOF systems. One can begin with an expression for the error in potential energy $\epsilon$

$$
\epsilon=E\left[\left(U(X)-\frac{1}{2} X^{T} K_{e} X\right)^{2}\right]
$$

where $U(X)$ is the potential energy of the original (nonlinear system) and $K_{e}$ is the stiffness matrix of the equivalent linear system.
A condition of minimized error $\epsilon \rightarrow \min$ requires the following

$$
E\left[\frac{\partial}{\partial K_{e i j}}\left(\left(U(X)-\frac{1}{2} X^{T} K_{e} X\right)^{2}\right)\right]=0 \quad i, j=1, N
$$

where $K_{e i j}$ are elements of matrix $K_{e}$. Omiting intermediate derivations $\Gamma$ one obtains the following system of $N^{2}$ linear equations with respect to unknown elements of matrix $K_{e}$ :

$$
\sum_{i, j=1}^{N} K_{e i j} E\left[x_{i} x_{j} x_{k} x_{l}\right]=2 E\left[x_{k} x_{l} U(X)\right]
$$

where $k, l=1, N$.

For exampleTequation (4) would have the following form for a two-degree-of-freedom system

$$
\begin{aligned}
& {\left[\begin{array}{cccc}
E\left[x_{1}^{4}\right] & E\left[x_{1}^{3} x_{2}\right] & E\left[x_{1}^{3} x_{2}\right] & E\left[x_{1}^{2} x_{2}^{2}\right] \\
E\left[x_{1}^{3} x_{2}\right] & E\left[x_{1}^{2} x_{2}^{2}\right] & E\left[x_{1}^{2} x_{2}^{2}\right] & E\left[x_{1} x_{2}^{3}\right] \\
E\left[x_{1}^{3} x_{2}\right] & E\left[x_{1}^{2} x_{2}^{2}\right] & E\left[x_{1}^{2} x_{2}^{2}\right] & E\left[x_{1} x_{2}^{3}\right] \\
E\left[x_{1}^{2} x_{2}^{2}\right] & E\left[x_{1} x_{2}^{3}\right] & E\left[x_{1} x_{2}^{3}\right] & E\left[x_{2}^{4}\right]
\end{array}\right]\left[\begin{array}{c}
K_{e 11} \\
K_{e 12} \\
K_{e 21} \\
K_{e 22}
\end{array}\right]} \\
& =2\left[\begin{array}{c}
E\left[x_{1}^{2} U(X)\right] \\
E\left[x_{1} x_{2} U(X)\right] \\
E\left[x_{1} x_{2} U(X)\right] \\
E\left[x_{2}^{2} U(X)\right]
\end{array}\right]
\end{aligned}
$$

Note that the 2 nd and 3 rd rows are identicalTthus an additional equation is required to solve this system. The additional equation(s) can be provided by the imposition of a condition of symmetry of the matrix $K_{e}$ :

$$
K_{e i j}=K_{e j i}
$$

The matrix of the system in equation (5) involves 4th order moments of displacements and the right-hand side (assuming that the potential energy is a function of the $2 \mathrm{nd} \Gamma 3 \mathrm{rd}$ and 4 th order terms) involves moments of 4 th 5 th and 6 th order. Using the Gaussian distributed $\Gamma$ zero-mean response assumption means that the odd order moments are zero and the higher even order statistical moments can be expressed in terms of the 2 nd order momentsTe.g. $\Gamma$

$$
\begin{gathered}
E\left[x_{i} x_{j} x_{k} x_{l}\right]=E\left[x_{i} x_{j}\right] E\left[x_{k} x_{l}\right]+E\left[x_{i} x_{k}\right] E\left[x_{j} x_{l}\right]+ \\
E\left[x_{i} x_{l}\right] E\left[x_{j} x_{k}\right]
\end{gathered}
$$

Therefore the matrix and right-hand side of (5) can be determined solely by the response covariance matrix. So the equivalent stiffness matrix at each iteration is determined through the use of response covariance terms from the previous iteration by solving equations (5). 


\section{Iterative scheme of equivalent linearization}

Having defined the equivalent linear stiffness matrix through either the force error or potential energy error minimization techniques Tone can proceed with the solution of the equivalent linear system. Assuming stationary excitation Ta stationary response is sought precluding the need for initial conditions. As the equivalent stiffness matrix $K_{e}$ is a function of the unknown displacement response vector $\Gamma$ the solution to the system of equations of motion takes an iterative form Ti.e.

$$
M \ddot{X}_{n+1}+C \dot{X}_{n+1}+\left(K+K_{e n}\right) X_{n+1}=F
$$

where new displacement response estimates are calculated from a system based upon the previous estimate and iterations are continued until a convergence criterion is satisfied.

The solution to the equivalent system in equation (7) for each iteration can be obtained in the frequency domain using the well known relation between the spectral density matrices for a linear system:

$$
S_{x}(\omega)_{n+1}=H_{n}(\omega) S_{f}(\omega) \bar{H}_{n}^{T}(\omega)
$$

where the over-bar indicates the complex-conjugate $\Gamma S_{f}$ is the spectral density matrix of the random excitation and the frequency response matrix is given by

$$
H_{n}(\omega)=\left[-\omega^{2} M+i \omega C+K+K_{e n}\right]^{-1}
$$

The zero-time-lag covariance matrix components participating in the matrix $K_{e n}$ are calculated from the response spectral density matrix using the WienerKhinchine formula

$$
E\left[x_{i} x_{j}\right]_{n}=\int_{-\infty}^{\infty}\left[S_{x i j}(\omega)\right]_{n} d \omega
$$

An implementation of the equivalent linearization approach outlined above was recently implemented in a special purpose in-house code to provide a tool for expedient study.

\section{Comparison with F-P-K Solutions}

The two equivalent linearization methods presented above will be compared with F-P-K solutions for SDOF and 2DOF systems.

\section{SDOF system}

Consider a SDOF system (Duffing oscillator):

$$
\ddot{q}(t)+2 \xi \omega_{0} \dot{q}(t)+\omega_{0}^{2} q(t)+\beta q^{3}(t)=f(t)
$$

where $q$ is a nondimensional coordinate/displacement. The addition to the potential energy originated from the nonlinear term is characterized by

$$
U(q)=\frac{1}{4} \beta q^{4}
$$

For this case ssolution of the system (5) (energy-based technique) provides the following equivalent stiffness

$$
k_{e}=2.5 \beta E\left[q^{2}\right]
$$

and equation (3) (force-base technique) yields

$$
k_{e}=3 \beta E\left[q^{2}\right]
$$

Comparison of response variances for this system versus the nonlinearity parameter $\frac{\beta}{\omega_{0}^{2}}$ is illustrated in Figure 1. A white noise excitation was taken as the input spectral density functionTi.e. $S_{f}(\omega)$ was constant and equal to $1.0 e+05$. The results correspond to an oscillator with a natural frequency of $57.4 \mathrm{~Hz}\left(\omega_{0}^{2}=1.301 \mathrm{e}+05 \mathrm{~s}^{-2}\right)$ and damping coefficient $\xi=0.005$. The three curves in Figure 1 correspond to the F-P-K solutionT force error minimization and energy error minimization versions.

Comparison of response variances for this oscillator versus the spectral density function value $S_{f}$ is illustrated in Figure $2 \Gamma$ where the nonlinearity parameter $\frac{\beta}{\omega_{0}^{2}}$ was fixed and equal to 10 . One can see that the energy error minimization version results are closest to the exact (F-P-K) solution results.

\section{DOF system}

As a next example consider the model of a 2DOF system in Figure 3. The equations of motion for this model have the form

$$
\begin{gathered}
m_{1} \ddot{q}_{1}+c_{1} \dot{q}_{1}+k_{1} q_{1}+k_{2}\left(q_{1}-q_{2}\right)+\beta_{1} q_{1}^{3}+\beta_{2}\left(q_{1}-q_{2}\right)^{3}=f_{1}(t) \\
m_{2} \ddot{q}_{2}+c_{2} \dot{q}_{2}+k_{2}\left(q_{2}-q_{1}\right)+\beta_{2}\left(q_{2}-q_{1}\right)^{3}=f_{2}(t)(10)
\end{gathered}
$$

The potential energy contribution from the nonlinear part is

$$
U\left(q_{1}, q_{2}\right)=\frac{1}{4} \beta_{1} q_{1}^{4}+\frac{1}{4} \beta_{2}\left(q_{2}-q_{1}\right)^{4}
$$

White noise was taken again as the input excitation with the spectral density matrix components: $S_{f 11}(\omega)=S_{f 22}(\omega)=1 \Gamma$ and $S_{f 12}(\omega)=S_{f 21}(\omega)=0$. The rest of the parameters of this model were as follows $m_{1}=m_{2}=1 \Gamma k_{1}=k_{2}=1 \Gamma c_{1}=c_{2}=0.1$ and $\beta_{1}=\beta_{2}=\beta$. A comparison of response variances versus the nonlinearity parameter $\beta$ for this $2 \mathrm{DOF}$ system (10) is shown in Figure 4. Again the energy-based version results are closer to the exact F-P-K solutionTthan the force-based version results.

Note that in the case of general MDOF nonlinear systems $\Gamma$ the determination of the expression for the potential energy can be complicated. This problem will be addressed in the section below. 


\section{Determination of Nonlinear Stiffness Coefficients}

So farTexamples were considered where the nonlinear stiffness coefficients were prescribed. In a general case of a MDOF system These coefficients have to be determined. One method of determining the nonlinear stiffness coefficients is through the use of a finite element approach. Existing finite element commercial programs are unable to provide these nonlinear stiffness coefficients directly. It is desirable to achieve a solution within a commercial finite element code to take advantage of the comprehensive element libraryTetc. necessary for modeling complex structures. This section describes a method of determining the nonlinear stiffness coefficients through the use of the nonlinear static solution capability that exists in many commercial finite element codes.

For MDOF structuresT it is expedient to seek a solution in modal coordinate space

$$
X=\Phi q
$$

where $\Phi$ is generally a subset $(L \leq N)$ of the linear eigenvectors (normal modes). Such a representation allows the size of the problem to be significantly reduced without a noticeable loss of accuracy in many cases.

One can obtain the following set of differential equations in terms of modal coordinates $q_{i}(i=1, L)$ :

$$
\ddot{q}_{i}(t)+\sum_{j=1}^{L} c_{i j} \dot{q}_{j}(t)+k_{i} q_{i}(t)+\gamma_{i}\left(q_{1}, q_{2}, \ldots, q_{L}\right)=f_{i}(t)
$$

where the nonlinear terms will be represented in the following form

$$
\gamma_{i}\left(q_{1}, q_{2}, \ldots, q_{L}\right)=\sum_{j, k=j}^{L} a_{j k}^{i} q_{j} q_{k}+\sum_{j, k=j, l=k}^{L} b_{j k l}^{i} q_{j} q_{k} q_{l}
$$

where the first index $j$ takes values 12 C.. IL The index $k$ takes values from $j$ (the current first index value) and up $j+1 \Gamma j+2 \ldots$ to $L$ and the third index $l$ takes values from $k$ (the current second index value) and up $k+1 \Gamma k+2$ to $L$.

The analytical form of the nonlinear terms facilitates the solution of equations (12) when the forces and displacements are random functions of time.

A procedure for determination of the coefficients $a_{j k}^{i}$ and $b_{j k l}^{i}$ is described briefly. This procedure requires the application of a finite element program with a nonlinear static solution capability. In this study MSC/PATRAN and MSC/NASTRAN programs 9,10 are utilized.

The suggested technique is based on the restoration of nodal applied forces from enforced nodal displacements prescribed to the whole structure in a static solution (linear and nonlinear). NamelyTby prescribing the physical nodal displacements (vector $X_{c}$ ) to the structureTone can restore the nodal forces $F_{T}$ and the corresponding nonlinear contribution $F_{c}$ :

$$
F_{c}=\Gamma\left(X_{c}\right)=F_{T}-K X_{c}
$$

The displacements $X_{c}$ can be prescribed by creating a displacement constraint set for the model in PATRAN $\Gamma$ then the nodal applied forces $F_{T}$ will arise as singlepoint-constraint forces in a NASTRAN nonlinear static solution.

To illustrate the technique one can begin with the prescription of displacements for the whole structure in the following form

$$
X_{c}=\phi_{1} q_{1}
$$

The nodal force vectors $F_{T}$ (nonlinear static solution) and $K X_{c}$ (linear static solution) are provided by NASTRAN. The nonlinear term $F_{c}$ can then be evaluated by equation (14). The vector of modal forces $\tilde{F}_{c}=\Phi^{T} F_{c}$ is calculated and it is represented as

$$
\begin{gathered}
\tilde{F}_{c}=\Phi^{T} F_{c}=\Phi^{T} \Gamma\left(X_{c}\right)=\Phi^{T} \Gamma\left(\phi_{1} q_{1}\right)= \\
{\left[a_{11}^{i}\right] q_{1} q_{1}+\left[b_{111}^{i}\right] q_{1} q_{1} q_{1}}
\end{gathered}
$$

where the sought stiffness coefficients $\left[a_{11}^{i}\right] \Gamma\left[b_{111}^{i}\right]$ are column-vectors $L \times 1(i=1, L)$. Note that all other nonlinear terms in (16) do not appear since $q_{j}=0$ for $j \neq 1$.

Prescribing a displacement field with opposite sign $X_{c}=-\phi_{1} q_{1}$ results in a modal force vector (denoted by $\left.\tilde{F}_{-c}\right)$ :

$$
\begin{gathered}
\tilde{F}_{-c}=\Phi^{T} F_{-c}=\Phi^{T} \Gamma\left(X_{c}\right)= \\
\Phi^{T} \Gamma\left(-\phi_{1} q_{1}\right)=\left[a_{11}^{i}\right] q_{1} q_{1}-\left[b_{111}^{i}\right] q_{1} q_{1} q_{1}
\end{gathered}
$$

where the quadratic (even) term will be the same as in (16) and the cubic (odd) term takes on a sign change.

Note that in the system of equations (16) and (17) $\Gamma$ the value of $q_{1}$ is given. The coefficients $\left[a_{11}^{i}\right] \Gamma\left[b_{111}^{i}\right]$ $(i=1, L)$ can be determined from this system of $2 \times L$ linear equations. In an analogous mannerTi.e. prescribing $X_{c}=\phi_{j} q_{j} \Gamma$ all other coefficients $\left[a_{j j}^{i}\right] \Gamma\left[b_{j j j}^{i}\right]$ can be determined.

A similar technique can be employed to determine coefficients with two or three inequal lower indicesTe.g. $\Gamma$ $\left[a_{12}^{i}\right]\left[\bar{b} b_{112}^{i}\right] \Gamma\left[b_{122}^{i}\right]$ or $\left[b_{123}^{i}\right]$. Note that coefficients of the latter type appear only if the number of retained eigenvectors $L$ in (11) is greater than or equal to 3 . Determination of coefficients $\left[a_{12}^{i}\right]\left[\left[b_{112}^{i}\right]\right.$ and $\left[b_{122}^{i}\right]$ will be considered as an example. Prescribe the displacement field to the model in the following form

$$
X_{e}=\phi_{1} q_{1}+\phi_{2} q_{2}
$$

then the calculated (using NASTRAN) corresponding modal force vector $\tilde{F}_{c}$ is represented as follows

$$
\tilde{F}_{c}=\Phi^{T} F_{c}=\Phi^{T} \Gamma\left(\phi_{1} q_{1}+\phi_{2} q_{2}\right)=
$$




$$
\begin{gathered}
{\left[a_{11}^{i}\right] q_{1} q_{1}+\left[b_{111}^{i}\right] q_{1} q_{1} q_{1}+\left[a_{22}^{i}\right] q_{2} q_{2}+\left[b_{222}^{i}\right] q_{2} q_{2} q_{2}+} \\
{\left[a_{12}^{i}\right] q_{1} q_{2}+\left[b_{112}^{i}\right] q_{1} q_{1} q_{2}+\left[b_{122}^{i}\right] q_{2} q_{2} q_{1}}
\end{gathered}
$$

Prescribing the opposite sign displacement field

$$
X_{c}=-\phi_{1} q_{1}-\phi_{2} q_{2}
$$

one obtains a second set of equations

$$
\begin{gathered}
\tilde{F}_{-c}=\Phi^{T} F_{-c}=\Phi^{T} \Gamma\left(-\phi_{1} q_{1}-\phi_{2} q_{2}\right)= \\
{\left[a_{11}^{i}\right] q_{1} q_{1}-\left[b_{111}^{i}\right] q_{1} q_{1} q_{1}+\left[a_{22}^{i}\right] q_{2} q_{2}-\left[b_{222}^{i}\right] q_{2} q_{2} q_{2}+} \\
{\left[a_{12}^{i}\right] q_{1} q_{2}-\left[b_{112}^{i}\right] q_{1} q_{1} q_{2}-\left[b_{122}^{i}\right] q_{2} q_{2} q_{1}}
\end{gathered}
$$

Summing (18) and (19)Tone obtains

$$
\tilde{F}_{c}+\tilde{F}_{-c}=2\left[a_{11}^{i}\right] q_{1} q_{1}+2\left[a_{22}^{i}\right] q_{2} q_{2}+2\left[a_{12}^{i}\right] q_{1} q_{2}
$$

From this equation $\Gamma$ the coefficients $\left[a_{12}^{i}\right]$ are determined (note that the coefficients $\left[a_{11}^{i}\right] \Gamma\left[a_{22}^{i}\right]$ were already determined above).

Now we have two sets of $L$ equations (18) and (19) $\Gamma$ but to determine cubic coefficients $\left[b_{112}^{i}\right]$ and $\left[b_{122}^{i}\right]$ from them is not possible since the system matrix has linearly dependent rows. Therefore $\Gamma$ an additional type of displacement field is required. One can prescribe the following type

$$
X_{a}=\phi_{1} q_{1}-\phi_{2} q_{2}
$$

Then the modal force vector is equal to

$$
\begin{gathered}
\tilde{F}_{a}=\Phi^{T} F_{a}=\Phi^{T} \Gamma\left(\phi_{1} q_{1}-\phi_{2} q_{2}\right)= \\
{\left[a_{11}^{i}\right] q_{1} q_{1}+\left[b_{111}^{i}\right] q_{1} q_{1} q_{1}+\left[a_{22}^{i}\right] q_{2} q_{2}-\left[b_{222}^{i}\right] q_{2} q_{2} q_{2}-} \\
{\left[a_{12}^{i}\right] q_{1} q_{2}-\left[b_{112}^{i}\right] q_{1} q_{1} q_{2}+\left[b_{122}^{i}\right] q_{2} q_{2} q_{1}}
\end{gathered}
$$

From the system of $2 * L$ linear equations (18) and $(20) \Gamma$ the coefficients $\left[b_{112}^{i}\right]$ and $\left[b_{122}^{i}\right]$ can be determined. In a similar mannerTall coefficients of the type $\left[b_{j j k}^{i}\right]$ and $\left[b_{k k j}^{i}\right]$ can be determined. A technique has been developed to determine all the coefficients $\left[a_{j k}^{i}\right] \Gamma\left[b_{j k l}^{i}\right]$ using a similar approach as above.

\section{Solution of modal equations}

Having the modal equations of motion (12) formulated $\Gamma$ solution to these equations can now be undertaken through a variety of techniques. For the case of random loading $\Gamma$ the application of the equivalent stochastic linearization was implemented in this study. Within the framework of the force-based technique Tthe equivalent stiffness matrix (according to the formula (3)) will have the following form

$$
K_{e}=E\left[\frac{\partial\left(\gamma_{1}, \gamma_{2}, \ldots, \gamma_{L}\right)}{\partial\left(q_{1}, q_{2}, \ldots, q_{L}\right)}\right]
$$

Note that the derivatives and expectations in (21) can be easily evaluated due to the analytical representation of the nonlinear terms in (13). A program producing the calculations described above has been developed and numerical results will be demonstrated in the next section.

Based upon the expressions derived in equation (13) $\Gamma$ one can proceed with the determination of potential energy $U$ in terms of modal coordinates. It is known that elastic force terms (linear + nonlinear) satisfy the following

$$
k_{i} q_{i}+\gamma_{i}\left(q_{1}, q_{2}, \ldots, q_{L}\right)=\frac{\partial U}{\partial q_{i}} \quad i=1, L
$$

Since all nonlinear coefficients in $\gamma_{i}\left(q_{1}, q_{2}, \ldots, q_{L}\right)$ have been determined $\Gamma$ the potential energy function $U\left(q_{1}, q_{2}, \ldots, q_{L}\right)$ can be derived and it can be used in the energy-based stochastic linearization technique. An implementation of the energy-based version for application to MDOF systems is considered as future work.

\section{Numerical Results for MDOF Structures}

It is important to note that the analysis of a vibrating structure in the nonlinear setting is necessary only if the comparison of two static solutions (linear and nonlinear ones for the highest deformation level) shows a noticeable difference in the displacement fields. To illustrate this $\Gamma$ one can consider the three beam structures shown in Fig.5a-c. It was found that for a cantilevered beam model (Fig. 5c) T the difference in terms of static flexural displacements is negligible. Two curves (Fig.6) corresponding to the linear and nonlinear models are indistinguishable Twhere the flexural displacement of the tip node versus the applied static base acceleration (inertial loading) is plotted. However for a beam in Fig. 5b and clamped-clamped beam (Fig.5a) the difference in displacements is quite noticeable (see Fig. 617) Tindicating that the vibration analysis should be conducted in the nonlinear setting.

The numerical results presented in this section correspond to models of the structures in Figures 5a and b. The results obtained with the SEMELRR DMAP are compared with the new method which employs the technique described above $\Gamma$ i.e. the determination of nonlinear stiffness coefficients plus the conventional (force-based) stochastic linearization technique.

SEMELRR was implemented in MSC/NASTRAN using equivalent linear modal degrees of freedom. This requires an eigensolution at each iteration $\Gamma$ but affords the most simple and versatile procedure readily adapted within the framework of the existing MSC/NASTRAN solution sequences. The original implementation was limited to spatially uniform mechanical loadsTbut has since been generalized to include spatial non-uniformity. The solution is also formulated to include the effects of static deflection due to mechanical or thermal loadsTmaterial nonlinearity and follower forces. Some work has been done to validate the prediction capability of SEMELRRTsee Robinson et al. ${ }^{7} \mathrm{~T}$ 


\begin{tabular}{|c|c|c|c|}
\hline material & $\begin{array}{c}\text { Young's } \\
\text { modulus }\end{array}$ & $\begin{array}{c}\text { Poisson's } \\
\text { ratio }\end{array}$ & density \\
\hline aluminum & $0.73 \mathrm{e}+11$ & 0.325 & $0.2763 \mathrm{e}+04$ \\
\hline $\begin{array}{c}\text { length } \\
\text { beam a) }\end{array}$ & $\begin{array}{c}\text { length } \\
\text { beam b) }\end{array}$ & width & thickness \\
\hline 0.4572 & 0.2286 & 0.0254 & 0.002261 \\
\hline
\end{tabular}

Table 1: Parameters of beams a) and b)

\begin{tabular}{|c|c|c|c|}
\hline$b_{111}^{1}$ & $b_{222}^{1}$ & $b_{112}^{1}$ & $b_{122}^{1}$ \\
\hline $0.899 \mathrm{e}+12$ & $0.977 \mathrm{e}+13$ & $0.191 \mathrm{e}+13$ & $0.139 \mathrm{e}+14$ \\
\hline$b_{111}^{2}$ & $b_{222}^{2}$ & $b_{112}^{2}$ & $b_{122}^{2}$ \\
\hline $0.638 \mathrm{e}+12$ & $0.608 \mathrm{e}+14$ & $0.139 \mathrm{e}+14$ & $0.293 \mathrm{e}+14$ \\
\hline
\end{tabular}

Table 2: Nonlinear stiffness coefficients for beam a)

but rigorous validation of all of its features and bounds of its applicability have not been fully established.

The parameters of the models in Fig.5a and $\mathrm{b}$ are shown in Table 1 (system of units is $\operatorname{SIC}[m] \Gamma\left[N / m^{2}\right] \Gamma$ $[\mathrm{kg}]$ etc.) $\Gamma$ where width and thickness are dimensions of the cross-section of the beams. The first two natural frequencies (associated with flexural modes in the excitation plane) for the beam in Fig.5a are $57.4 \mathrm{~Hz}$ and $310.1 \mathrm{~Hz}$ and the first two natural frequencies for the beam in Fig. $5 \mathrm{~b}$ are $35.6 \mathrm{~Hz}$ and $220 \mathrm{~Hz}$. In all cases the first two (symmetric for clamped-clamped beam) flexural mass-normalized modes were chosen to approximate the motion of beams according to formula (11).

The nonlinear stiffness coefficients determined with application of the procedure described above are summarized in Tables 2 and 3. The quadratic terms were negligible 5 so only the $3 r d$ order terms are shown. Since the modal coordinates $q_{1}, q_{2}$ are nondimensional $\Gamma$ the units of these nonlinear coefficients are in $[N * m]$.

Note that from (22) would follow that

$$
\frac{\partial \gamma_{j}}{\partial q_{k}}=\frac{\partial \gamma_{k}}{\partial q_{j}}=\frac{\partial^{2} U}{\partial q_{k} q_{j}}
$$

and comparing the terms with like powers in $q_{j}$ and $q_{k}$ leads to a certain relation between the nonlinear coefficients $\Gamma$ for example $\Gamma$ for the cubic coefficients $b_{122}^{1}$ and $b_{112}^{2}$ it is

$$
b_{122}^{1}=b_{112}^{2}
$$

and for other typesTit is

$$
3 b_{222}^{1}=b_{122}^{2} \quad 3 b_{111}^{2}=b_{112}^{1}
$$

It turned out that the computed nonlinear stiffness coefficients (see Tables 2 and 3 ) are in an excellent agreement with these relations.

The results in terms of the RMS displacement of the middle and tip nodes (Fig. 5a and b) are shown in

\begin{tabular}{|c|c|c|c|}
\hline$b_{111}^{1}$ & $b_{222}^{1}$ & $b_{112}^{1}$ & $b_{122}^{1}$ \\
\hline $0.359 \mathrm{e}+13$ & $-0.713 \mathrm{e}+14$ & $-0.233 \mathrm{e}+14$ & $0.722 \mathrm{e}+14$ \\
\hline$b_{111}^{2}$ & $b_{222}^{2}$ & $b_{112}^{2}$ & $b_{122}^{2}$ \\
\hline$-0.779 \mathrm{e}+13$ & $0.247 \mathrm{e}+15$ & $0.722 \mathrm{e}+14$ & $-0.213 \mathrm{e}+15$ \\
\hline
\end{tabular}

Table 3: Nonlinear stiffness coefficients for beam b)

Fig. 8 and 9. A vertical base white noise excitation (acceleration $a_{b}$ ) provided inertial loading which was spread over a $20-320 \mathrm{~Hz}$ range. One can see that numerical results produced with the SEMELRR code and the new method differ by about $20 \%$ for the case of clamped-clamped beam. The difference is about $30 \%$ for beam b). In each case the SEMELRR's RMS displacements are less than the RMS displacements from the new approach.

Unfortunately there are no exact solutions available for these structuresT so comparisons are not possible. HoweverTrecent experimental measurements (not presented here) indicate that the new method predicts RMS responses more in agreement with their physical counterparts than the SEMELRR solution sequence. This will be quantified with further numerical and experimental work.

\section{Summary}

The energy-based version of stochastic linearization technique has been extended to MDOF systems and the numerical results have shown superior performance of this technique in comparison with the conventional linearization version.

A new method for determination of nonlinear stiffness coefficients has been suggested and applied to several examples of beam-like structures. This method has been incorporated into a program which calculates a steady-state response of a MDOF structure to a Gaussian zero-mean excitation. Efforts are presently underway to implement this capability into MSC/NASTRAN through a DMAP Alter.

Some difference (about 20-30\% range) has been found between the two independent results in terms of prediction of nonlinear response. Further numerical studies and experimental work will be devoted to this problem.

\section{$\underline{\text { References }}$}

[1] Bolotin V.V.TStatistical methods in structural mechanics THolden-DayГInc. Г1969.

[2] Lin Y.K.TProbabilistic theory of structural dynam-

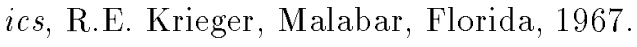

[3] Roberts J.B.TSpanos P.D.TRandom vibration and stochastic linearization T John Wiley and SonsT 1990 . 


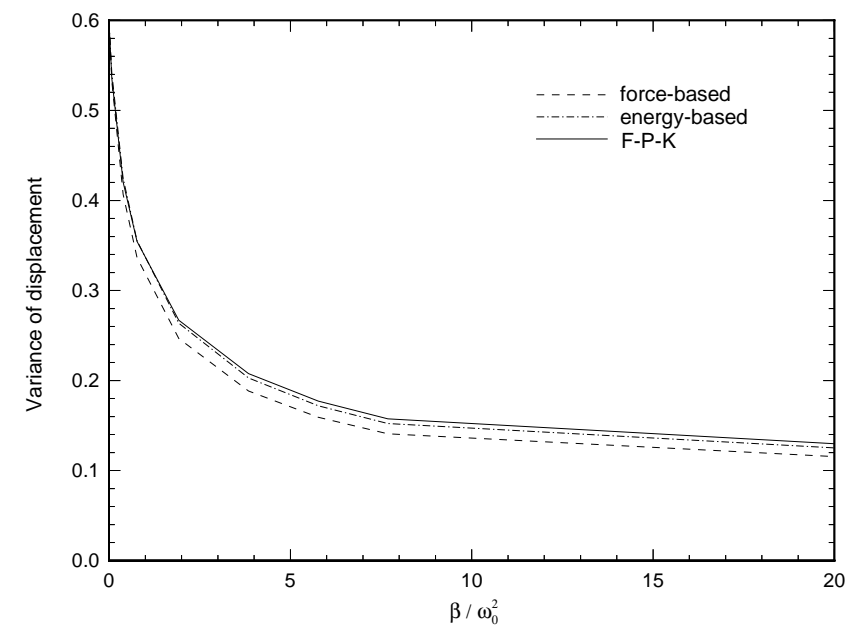

Figure 1: Variance of displacement vs. nonlinearity parameter for the SDOF system with natural frequency $\omega_{0} / 2 \pi=57.4 \mathrm{~Hz}$ and damping coefficient $\xi=0.005$

[4] Atalik T.S.T S. UtkuT "Stochastic linearization of multi-degree-of-freedom non-linear systems" Earthquake engineering and structural dynamics, Vol. 4ГJuneГ1976Грp. 411-420.

[5] Elishakoff I.T Zhang R.T "Comparison of new energy-based versions of the stochastic linearization technique" $\Gamma$ Nonlinear Stochastic Mechanics, N.Bellomoto, F.Casciati (Eds.)ГIUTAM Symposium ГTurinTSpringer-Verlag T1992 Tpp.201-211.

[6] Zhang X.T Elishakoff I.T Zhang R.T"A stochastic linearization technique based on minimum mean square deviation of potential energy" $\Gamma$ Stochastic structural dynamics, Y.K. Lin, I. Elishakoff (Eds.)Г Boca RatonIFloridaT SpringerVerlagГ1991Гpp. 328-338.

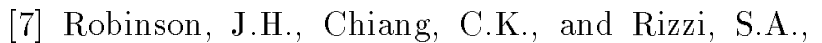
"Nonlinear Random Response Prediction Using MSC/NASTRAN"T NASA TM 109029T October 1993.

[8] MSC/PATRAN release 7.5T The MacNeal Schwendler Corporation 1997.

[9] MSC/NASTRAN QUICK REFERENCE GUIDE, version 70.5T The MacNeal-Schwendler Corporation $\Gamma 1998$.

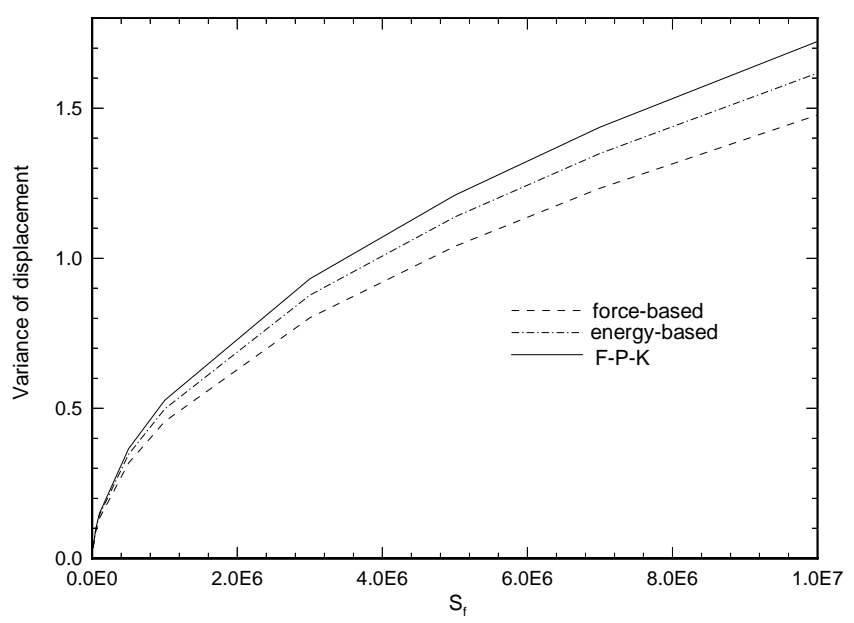

Figure 2: Variance of displacement vs. spectral density of force for the SDOF system with natural frequency $\omega_{0} / 2 \pi=57.4 H z \Gamma$ damping coefficient $\xi=0.005$ and $\beta / \omega_{0}^{2}=10$

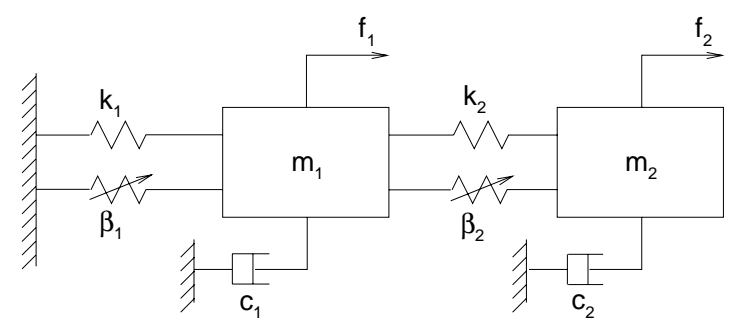

Figure 3: Example of a two-degree-of-freedom system 


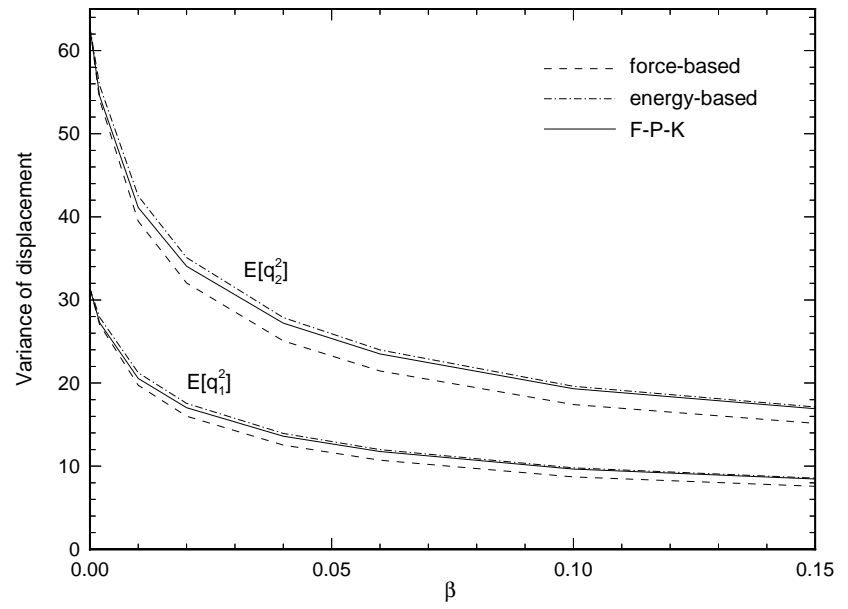

Figure 4: Variancies of displacement vs. nonlinearity parameter for the 2DOF system

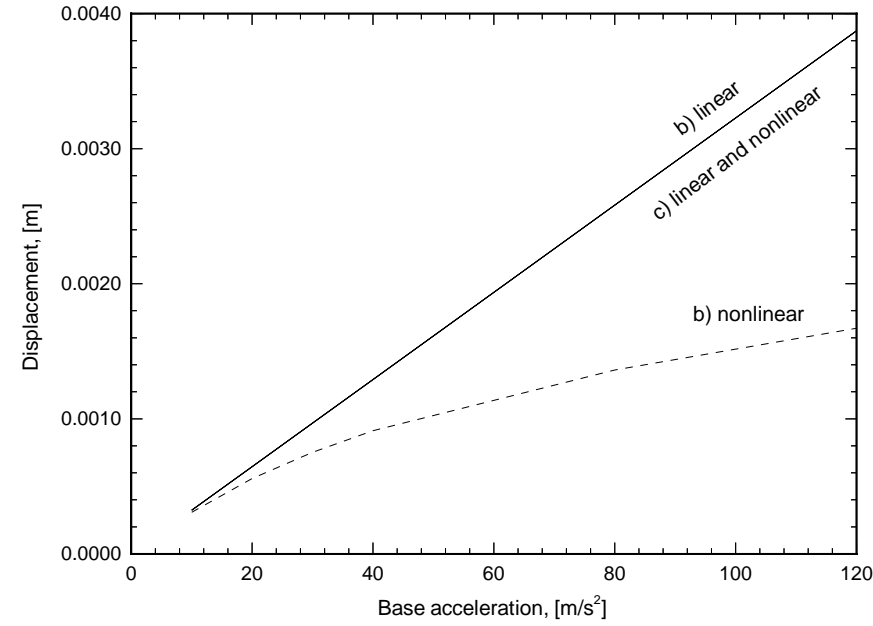

Figure 6: Displacement as a function of inertial load beams in Fig. $5 \mathrm{bTc}$ $a_{0} \mid$

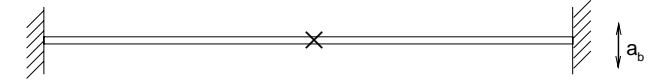

$a_{b}$

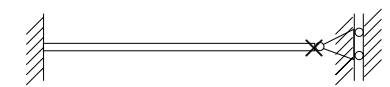

$\left.a_{b}\right)$

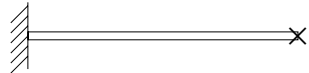

a)

b)

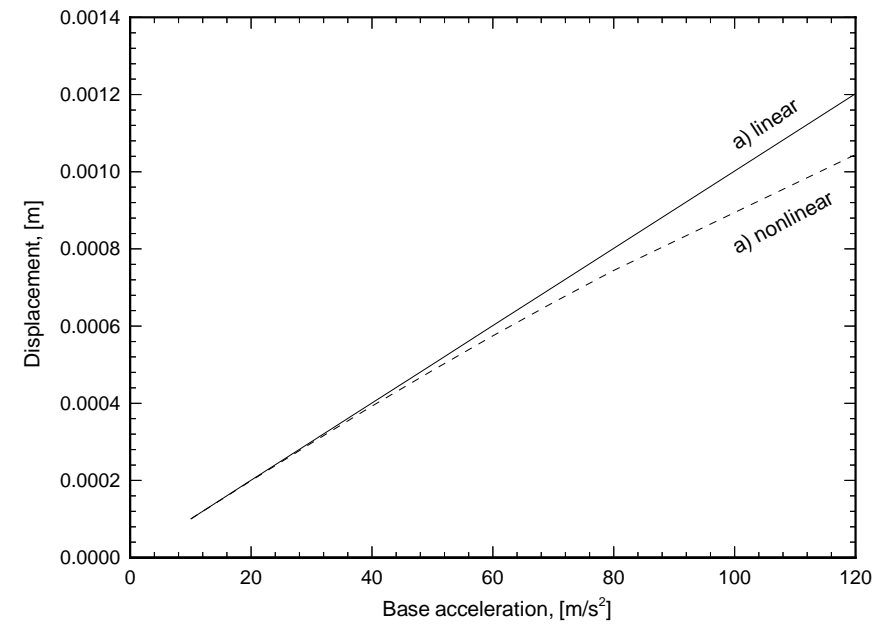

Figure 7: Displacement as a function of inertial load beam in Fig.5a 


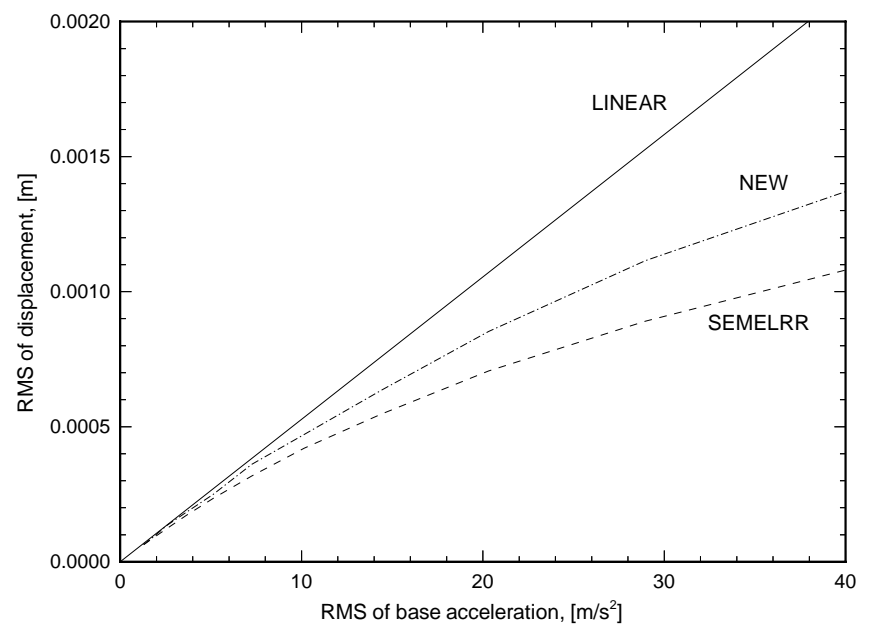

Figure 8: RMS displacement for beam in Fig. 5a as a function of inertial random loading

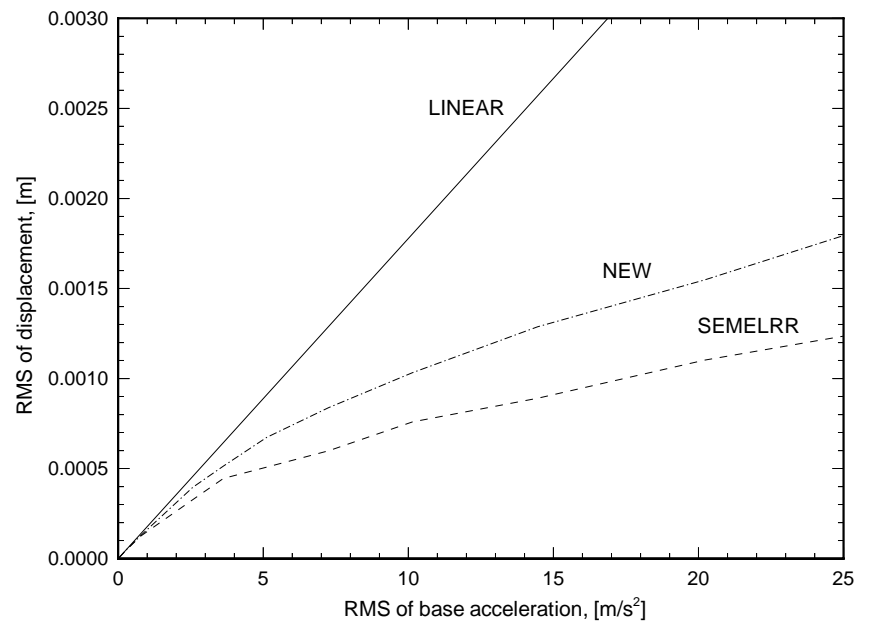

Figure 9: RMS displacement for beam in Fig. 5b as a function of inertial random loading 